\title{
Study of upper airway inflammation in patients with obstructive sleep apnea-hypopnea syndrome
} Enas E. Mohamed ${ }^{\mathrm{a}}$, Ayman I. Baess ${ }^{\mathrm{a}}$, Yasser M. Eldowik ${ }^{\mathrm{b}}$

\author{
Background Pharyngeal lavage was reported as a novel \\ technique for noninvasive assessment of inflammation of the \\ pharynx.
}

Aim To study upper airway inflammation in patients with obstructive sleep apnea-hypopnea syndrome (OSAHS) and its correlation with systemic inflammation.

Patients and methods A total of 36 patients with known OSAHS, admitted to the Chest Department, Alexandria Main University Hospital (group I), and 15 healthy volunteers (group II) were enrolled into the study. Informed consent was taken from all participants. The two groups underwent complete history taking, assessment of BMI, measurement of serum C-reactive protein (CRP), overnight polysomnography, and oropharyngeal lavage (OPL) analysis.

Results In group I, the most prevalent cell type of OPL was lymphocytes followed by neutrophils. Compared with control group (group II), the most prevalent cell type in OPL was macrophages followed by epithelial cells. Regarding the OPL differential cell count which denotes local pharyngeal inflammation, there was a statistically significant difference between both groups $(P<0.001)$. Similarly, CRP, a marker of systemic inflammation, showed a significant statistical difference between both groups $(P<0.001)$. Strikingly, there was no statistically significant correlation between CRP and

\section{Introduction}

Obstructive sleep apnea-hypopnea syndrome (OSAHS) is a disorder characterized by frequent episodes of obstruction in the upper airway during sleep [1]. Evidence advocates that both local airway inflammation and systemic inflammation are involved in the pathophysiology of this apparently mechanical disorder [2,3]. Leukocytic infiltration is markedly increased in the mucosa of the upper airway of adults with OSAHS [4]. Accumulating evidence suggests that local and systemic inflammatory responses also exist in patients with OSAHS [5]. Creactive protein (CRP) levels are raised in children with OSAHS [6,7]. Recently, Vicente et al reported a novel technique for noninvasive examination of pharyngeal inflammation called oropharyngeal lavage (OPL) [8].

\footnotetext{
Aim

The aim of this work was to study upper airway inflammation in patients with OSAHS and its correlation with systemic inflammation.
}

\section{Patients and methods}

Study population and patients

A total of 36 patients with known OSAHS, admitted to the Chest Department, Alexandria Main University apnea-hypopnea index $(P=0.604)$. Snoring, apnea-hypopnea index, and other sleep parameters correlated significantly with lymphocytic predominance in OPL $(P<0.001)$, whereas CRP correlates significantly with neutrophils and eosinophil's in OPL $(P<0.001$ and $P=0.015$, respectively).

Conclusion Our study had provided a novel vision into the pathophysiology of OSAHS in emphasizing the existence of upper airway lymphocytic inflammation. OPL is a simple, applicable, and easy noninvasive procedure for assessment of upper airway inflammation.

Egypt J Bronchol 2019 13:754-759

(C) 2020 Egyptian Journal of Bronchology

Egyptian Journal of Bronchology 2019 13:754-759

Keywords: local inflammation, oropharyngeal lavage, obstructive sleep apnea-hypopnea syndrome

aDepartment of Chest Diseases, Alexandria University, Alexandria,

bepartment of Pathology, Al-Azhar University, Cairo, Egypt

Correspondence to Dr. Ayman I. Baess, MD, PhD, Department of Chest Diseases, Faculty of Medicine, Alexandria University, Alexandria, 21131, Egypt. Tel: +20 100682 2068; fax: +20 3485 3961; e-mails: ayman.baeis@yahoo.com, ayman.baeis@alexmed.edu.eg

Received: 22 March 2019 Accepted: 30 March 2019 Published: 21 January 2020

Hospital (group I), and 15 healthy volunteers (group II) were enrolled into the study. All patients and subjects were prospectively enrolled after obtaining informed consents during the period of May 2017 through August 2018. The study was approved by the Local Institutional Ethics Committee of the Faculty of Medicine, Alexandria University.

\section{Study measurements}

Exclusion criteria

Patients with severe associated diseases (e.g. liver failure, heart failure, kidney failure, or any neuromuscular disorders), acute or chronic inflammatory disease of the airways [e.g. chronic obstructive pulmonary disease (COPD)], or use of any drugs that may affect the sleep pattern (e.g. sedatives or anxiolytics) were excluded from this study.

All patients were subjected to the following:

(1) Full history taking including age, sex, and history of other diseases.

This is an open access journal, and articles are distributed under the terms of the Creative Commons Attribution-NonCommercial-ShareAlike 4.0 License, which allows others to remix, tweak, and build upon the work non-commercially, as long as appropriate credit is given and the new creations are licensed under the identical terms. 
(2) Assessment of BMI, which was calculated as weight/height $\left(\mathrm{kg} / \mathrm{m}^{2}\right)$.

(3) Full clinical examination, including general, local chest, and throat examination.

(4) Measuring serum CRP.

(5) Sleep study: Standard full PSG study was conducted with the SOMNOscreen System (SOMNOmedics GmbH; Am Sonnenstuhl, Randersacker, Germany). The following parameters were included: flow (cannula and/or thermistor), oxygen saturation, snore (cannula and/or microphone), plethysmogram, thoracic movement, abdominal movement, periodic leg movement, pulse rate, ECG, electrooculogram, electroencephalogram, and continuous positive airway pressure (CPAP)/BiPAP pressure. The polysomnographic evaluation was done automatically then combined with manual scoring. The analysis included the following:

(a) Apnea-hypopnea index (AHI): severity of OSAHS was classified according to the following: AHI 5 to less than $15 / \mathrm{h}$ mild, AHI 15 to less than $30 / \mathrm{h}$ moderate, AHI more than $30-50 / \mathrm{h}$ severe, and more than $50 / \mathrm{h}$ very severe.

(b) Oxygen desaturation index 4\% (ODI 4\%): ODI of more than $4 \%$.

(c) Time of sleep with oxygen saturation below $90 \%$.

(6) OPL analysis: the samples were taken in the morning after waking up from sleep. All patients and the healthy controls rinsed their mouth with sterile $0.9 \%$ saline solution at room temperature. Next they gargled with $50 \mathrm{ml}$ of sterile $0.9 \%$ saline solution in several portions for $5-10 \mathrm{~s}$ each one. For sample collection, they spat the saline into a sterile container. Cell counting was accomplished by a skilled investigator. The OPL was prepared on thin smear cytology slides and stained by $\mathrm{H} \& \mathrm{E}$ stain. Under $\times 10$ magnification, the slide was scanned to ensure even distribution of cells. Under $\times 40$ magnification in $10 \mathrm{Hpf}$, the differentiated cells in 100 cells were counted, and the total differentiated count for each cell and percentage of each was assessed.

\section{Statistical analysis}

All statistical analyses were shown using IBM SPSS software package (IBM SPSS statistics for Windows, version 20.0.; IBM Corp., Armonk, New York, USA). Qualitative data were described using number and percent, whereas normally quantitative data were expressed in mean $\pm \mathrm{SD}$, and abnormally distributed data were expressed in median (interquartile range). Comparison between different groups regarding categorical variables was tested using $\chi^{2}$-test. If normal data distribution existed, parametric tests were applied. If the data were abnormally distributed, nonparametric tests were used. For normally distributed data, comparison between the two studied groups was done using independent $t$-test. For normally distributed data, comparison between more than two populations was analyzed by $F$-test (analysis of variance) and post-hoc test (least significant difference). For abnormally distributed data, comparison between two independent population was done using Mann-Whitney test, whereas Kruskal-Wallis test was used to compare between different groups, and pairwise comparison was assessed using Mann-Whitney test. Correlations between two quantitative variables were assessed using spearman coefficients regarding normality of the data. Significant results were detected at the level of $P$ value less than 0.05 .

\section{Results}

A total of 36 patients with documented OSAHS (group I), admitted to the Chest Department, Alexandria Main University Hospital, and 15 healthy volunteers (group II) were included in this study after obtaining informed consents.

Patients in both groups were matched regarding age and sex (Table 1). Regarding anthropometric measures, namely, weight, height, and BMI, there was a statistically significant difference between both groups $(P=0.036$; Table 1$)$.

In group I, the most prevalent cell type of OPL was lymphocytes followed by neutrophils. Compared with control group (group II), the most prevalent cell types in OPL were macrophages followed by epithelial cells. Regarding the OPL differential cell count, which denotes local pharyngeal inflammation, there was a statistically significant difference between both groups $(P<0.001)$. Similarly, CRP, a marker of systemic inflammation, showed a significant statistical difference between both groups $(P<0.001$; Table 1$)$.

Regarding the sleep laboratory parameters, namely, snoring, AHI, T90, and ODI 4\%, there was a significant statistical difference between both groups $(P<0.001)$. Both groups were matched for comorbidities, namely, hypertension and diabetes (Table 1).

Table 2 shows the correlation between AHI, T90, ODI $4 \%$, and CRP with different parameters in group I 
Table 1 Comparison between the two studied groups according to different parameters

\begin{tabular}{|c|c|c|c|c|}
\hline & Cases $(n=36)$ & Control $(n=15)$ & Test of Significance & $P$ \\
\hline \multicolumn{5}{|l|}{ Sex } \\
\hline Female & $20(55.6)$ & $6(40)$ & $\chi^{2}=1.025$ & 0.311 \\
\hline Male & $16(44.4)$ & $9(60)$ & & \\
\hline Age & $51.8 \pm 5.4$ & $50.8 \pm 4.7$ & $t=0.645$ & 0.522 \\
\hline Weight & $124.4 \pm 23$ & $94.2 \pm 9.2$ & & \\
\hline Height & $163.3 \pm 4.4$ & $172.6 \pm 7.1$ & $t=0.025$ & $0.036^{*}$ \\
\hline BMI & $46.7 \pm 8.89$ & $31.8 \pm 5.64$ & & \\
\hline \multicolumn{5}{|c|}{ Lavage differential cell count } \\
\hline Neutrophils & $11(5-19.3)$ & $2(1-3)$ & $\mathrm{U}=33.50^{\star}$ & $<0.001^{*}$ \\
\hline Lymphocytes & $18(10-34.8)$ & $9(7-10)$ & $U=93.50$ & $<0.001^{*}$ \\
\hline Eosinophil's & $0.5(0-2)$ & $0(0-1)$ & $U=221.0$ & 0.269 \\
\hline Macrophages & $0.5(0-12.5)$ & $77(75-78)$ & $U=0.00^{*}$ & $<0.001^{*}$ \\
\hline Epithelial cells & $54(40-70)$ & $13(10-14)$ & $U=37.0$ & $<0.001^{*}$ \\
\hline CRP & $14(11.3-22.5)$ & $4(3-6)$ & $U=21.0^{*}$ & $<0.001^{*}$ \\
\hline \multicolumn{5}{|l|}{$\mathrm{AHI}$} \\
\hline Median (25-75) & $22.5(13.0-35.0)$ & $2.0(1.0-4.0)$ & $U=0.0^{*}$ & $<0.001^{*}$ \\
\hline Mean $\pm S D$ & $24.4 \pm 12.9$ & $2.4 \pm 1.9$ & & \\
\hline \multicolumn{5}{|l|}{ T90 } \\
\hline Median (25-75) & $22.5(13.0-43.5)$ & $1.0(0.0-1.0)$ & $U=0.0^{*}$ & $<0.001^{*}$ \\
\hline Mean \pm SD & $26.7 \pm 15.3$ & $0.5 \pm 0.5$ & & \\
\hline \multicolumn{5}{|l|}{ ODI $4 \%$} \\
\hline Median (25-75) & $19(12.0-44.8)$ & $1.0(0.0-1.4)$ & $U=0.0^{*}$ & $<0.001^{*}$ \\
\hline Mean \pm SD & $25.6 \pm 17.1$ & $0.8 \pm 0.7$ & & \\
\hline \multicolumn{5}{|l|}{ Snoring } \\
\hline Nonsnorer & $10(27.8)$ & $12(80)$ & $\chi^{2}=13.138^{*}$ & $0.001^{*}$ \\
\hline Mild snorer & $13(36.1)$ & $3(20)$ & & \\
\hline Severe snorer & $13(36.1)$ & 0 & & \\
\hline \multicolumn{5}{|l|}{ DM } \\
\hline Negative & $15(41.7)$ & 5 (33.3) & $\chi^{2}=0.308$ & 0.579 \\
\hline Positive & $21(58.3)$ & $10(66.7)$ & & \\
\hline \multicolumn{5}{|l|}{ HTN } \\
\hline Negative & $13(36.1)$ & 5 (33.3) & $\chi^{2}=0.036$ & 0.850 \\
\hline Positive & $23(63.9)$ & $10(66.7)$ & & \\
\hline
\end{tabular}

Qualitative data were described using $n(\%)$, whereas normally quantitative data were expressed in mean $\pm \mathrm{SD}$, and abnormally distributed data were expressed in median (interquartile range). AHI, apnea-hypopnea index; CRP, C-reactive protein; DM, diabetes mellitus; HTN, hypertension; ODI $4 \%$, oxygen desaturation index more than $4 \%$; T90, time of sleep with oxygen saturation below $90 \%$. $P$ : $P$ value for comparing between the two studied groups. ${ }^{*} P \leq 0.05$, statistically significant.

(cases). Strikingly, there was no statistically significant correlation between CRP and AHI $(P=0.604)$. Snoring, AHI, T90, and ODI $4 \%$ correlated significantly with lymphocytic predominance in OPL $(P<0.001$; Fig. 1), whereas CRP correlates significantly with neutrophils and eosinophil's in OPL $(P<0.001$ and $P=0.015$, respectively; Fig. 2). Table 3 shows the relation between snoring severity and differential cell count in OPL.

\section{Discussion}

The assessment of inflammation existing in patients with OSAHS at the main source of airway obstruction (upper airway) and its relation to systemic consequences was accomplished using a novel procedure. The OPL as a technique is simple, easy, and noninvasive; besides, it estimates the local upper airway effects of recurrent collapse and snoring throughout repetitive cycles in patients with OSAHS. Systemic inflammation might add to weakening of the respiratory muscles, disturbed respiratory centers control, and harming the protective mechanisms that maintain airway patency in these patients $[9,10]$.

In this study, we found that the most prevalent cell type of OPL in OSAHS group was lymphocytes followed by neutrophils, with significant difference between cases and control groups. This matched with the results of Sekosan et al. [3] and by Boyd et al. [4] who demonstrated leukocyte infiltration and lymphocytic inflammatory cells in the mucosa of upper airway in patients with OSAHS. Furthermore, Hauber et al. [11] evaluated the OPL as a new technique to investigate inflammation of the mucosa of the pharynx in patients 
Table 2 Correlation between apnea-hypopnea index, time of sleep with oxygen saturation below $90 \%$, oxygen desaturation index more than $4 \%$, and C-reactive protein with different parameters in cases group

\begin{tabular}{|c|c|c|c|c|c|c|c|c|c|c|}
\hline & \multicolumn{2}{|c|}{$\mathrm{AHI}$} & \multicolumn{2}{|c|}{ T90 } & \multicolumn{2}{|c|}{ ODI $4 \%$} & \multicolumn{2}{|c|}{ CRP } & \multicolumn{2}{|c|}{ Snoring } \\
\hline & $r_{\mathrm{s}}$ & $P$ & $r_{\mathrm{s}}$ & $P$ & $r_{\mathrm{s}}$ & $P$ & $r_{\mathrm{s}}$ & $P$ & $r_{\mathrm{s}}$ & $P$ \\
\hline $\mathrm{AHI}$ & - & - & $0.860^{*}$ & $<0.001$ & $0.832^{*}$ & $<0.001$ & 0.089 & 0.604 & $0.574^{\star}$ & $<0.001$ \\
\hline Neutrophils (\%) & 0.148 & 0.390 & 0.121 & 0.482 & 0.103 & 0.548 & $0.805^{*}$ & $<0.001$ & $0.454^{\star}$ & 0.001 \\
\hline Lymphocytes (\%) & $0.896^{\star}$ & $<0.001$ & $0.894^{*}$ & $<0.001$ & $0.895^{\star}$ & $<0.001$ & 0.038 & 0.826 & $0.657^{*}$ & $<0.001$ \\
\hline Eosinophil's (\%) & 0.060 & 0.726 & -0.100 & 0.563 & -0.087 & 0.613 & $0.404^{*}$ & 0.015 & 0.158 & 0.269 \\
\hline Macrophages (\%) & 0.069 & 0.690 & -0.032 & 0.854 & -0.110 & 0.522 & -0.116 & 0.501 & $-0.442^{*}$ & 0.001 \\
\hline Epithelial cells (\%) & $-0.603^{*}$ & $<0.001$ & $-0.428^{*}$ & 0.009 & $-0.376^{*}$ & 0.024 & $-0.577^{\star}$ & $<0.001$ & 0.235 & 0.096 \\
\hline
\end{tabular}

$\mathrm{AHI}$, apnea-hypopnea index; CRP, C-reactive protein; ODI 4\%, oxygen desaturation index more than $4 \%$; $r_{\mathrm{s}}$, Spearman coefficient; T90, time of sleep with oxygen saturation below $90 \%$. ${ }^{*} P \leq 0.05$, statistically significant.

Figure 1

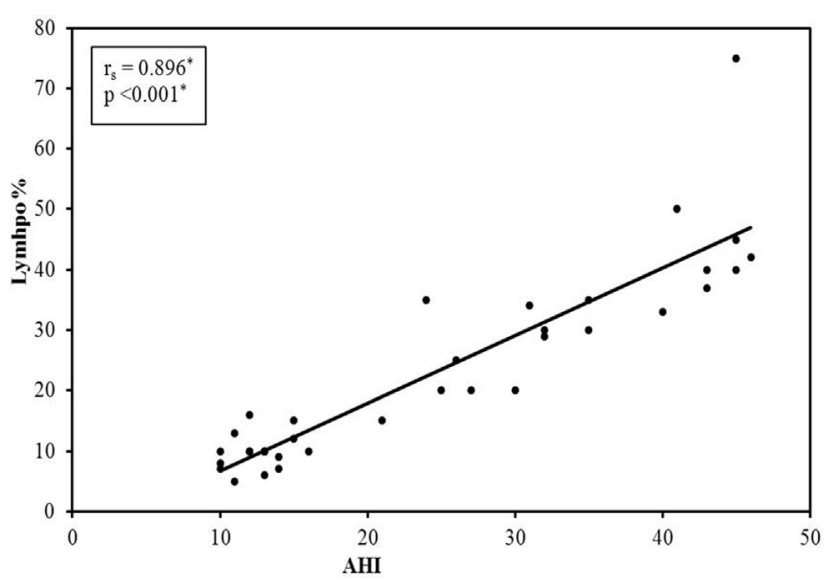

Correlation between apnea-hypopnea index (AHI) and oropharyngeal lavage (OPL) lymphocytes.

with sleep-related breathing disorders. They stated that patients with OSAHS had markedly significant numbers of lymphocytes in comparison with the control group patients. Patients having moderate or severe OSAHS had considerably higher quantities of lymphocytes compared with mild OSAHS cases, with statistical difference $(P<0.05)$. Lymphocytic count was higher in snorer than nonsnorer. It is not obvious whether local upper airway inflammation in OSAHS is the cause or the result of pharyngeal collapse. Consequently, an animal model study of frequent airway collapse established the theory that mechanical trauma occurring owing to airway collapse led to upper airway mucosal inflammation [12]. Snoring is known as a first phase in the progress of OSAHS. Our data agreed with those studies and suggest that upper airway lymphocytic mucosal inflammation could be an essential component. Additional probability is that snoring and OSAHS symbolize different categories of sleep-related breathing disorders. Extensive follow-up research studies of snorers can support and explain this issue.

Vicente et al. [8] revealed a high level of interleukins (ILs) mainly IL-6 and IL-8 $(P<0.05)$, although the total
Figure 2

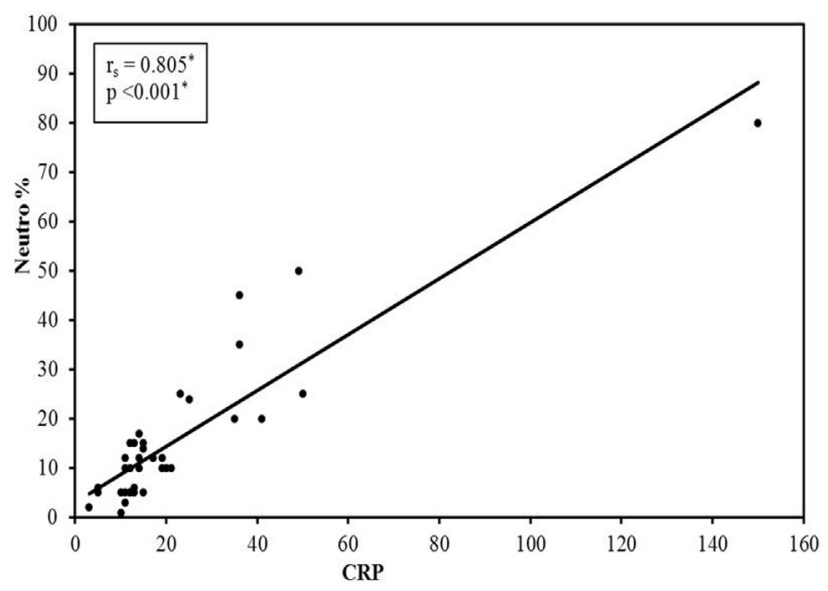

Correlation between C-reactive protein (CRP) and oropharyngeal lavage (OPL) neutrophils.

lymphocytic count, \%T lymphocytes, and \%CD4+ were higher $(P<0.05)$ compared with the control group. Moreover, there were no differences between patients and control group in plasma levels of the inflammatory biomarkers as IL-6 and 8, tumour necrosis factor (TNF) $-\alpha$, and CRP or lymphocytic count. No correlation for any inflammatory marker was achieved between OPL and plasma. During the follow-up of patients treated with CPAP, a reduction was found in the pharyngeal inflammatory markers with no alteration with plasma inflammatory markers. Vicente et al. [8] found that lymphocyte percentage was higher in severe OSAHS group compared with the control $(P<0.05)$ and the simple snorer group $(P<0.05)$. CD4+ was the main $\mathrm{T}$-cell subclass and was significantly higher in severe OSAHS (55-63\%) compared with both control and simple snorer groups $(P<0.001$ and $<0.05)$. These alterations were predominantly caused by activated $\mathrm{T}$ lymphocytes. Patients on CPAP therapy revealed a reduction in the OPL inflammatory cells, which was significant for total leukocytes, percentage of lymphocytes, and percentage of CD4+ T-cells $(P=0.034,0.008$, and 0.02 , respectively). Similar changes in the biomarkers were found after one-year 
Table 3 Relation between snoring and different parameters in group I 'cases'

\begin{tabular}{lccccc}
\hline & \multicolumn{3}{c}{ Snoring } & $H$ & $P$ \\
\cline { 2 - 4 } & Nonsnorer $(n=10)$ & Mild snorer $(n=13)$ & Severe snorer $(n=13)$ & & \\
\hline Neutrophils & $5.0(5.0-12.5)$ & $15.0(10.0-30.0)$ & $12.0(5.5-15.5)$ & 5.358 & 0.069 \\
Lymphocytes & $10.0^{\mathrm{b}}(6.8-11.3)$ & $15.0^{\mathrm{a}, \mathrm{b}}(10.0-32.0)$ & $33.0^{\mathrm{a}}(20.0-38.5)$ & $12.300^{*}$ & $0.002^{*}$ \\
Eosinophil's & $0.0^{\mathrm{b}}(0.0-1.0)$ & $2.0^{\mathrm{a}}(0.0-5.0)$ & $0.0^{\mathrm{a}, \mathrm{b}}(0.0-1.5)$ & $6.307^{*}$ & $0.043^{\star}$ \\
Macrophages & $5.0(0.0-20.0)$ & $0.0(0.0-3.0)$ & $1.0(0.0-12.5)$ & 2.027 & 0.363 \\
Epithelial cells & $74.0(44.5-86.0)$ & $50.0(40.0-60.0)$ & $43.0(39.0-39.0)$ & 3.743 & 0.154 \\
\hline
\end{tabular}

Abnormally distributed data were expressed in median (interquartile range). Means with common letters are not significant (i.e. means with different letters are significant). $H: H$ for Kruskal-Wallis test, pairwise comparison between each two groups was done using post-hoc test (Dunn's for multiple comparisons test). $P$ : $P$ value for comparing between the studied groups. ${ }^{*} P \leq 0.05$, statistically significant.

follow-up; again the reduction in local inflammatory markers was in OPL but not in plasma. However, Li et al. [13] established the association between inflammation of the upper airway and severity of OSAHS in children. Children with OSAHS had significantly higher percentage of sputum neutrophil than those without OSAHS $(P=0.006)$, and the percentage of sputum neutrophil was significantly associated with OSAHS $(P=0.013)$.

In this study, CRP, a biomarker of systemic inflammation, showed a significant statistical difference between both groups, although we found no statistically significant correlation between CRP and AHI $(P=0.604)$. Elevated measurement of systemic markers of inflammation such as CRP had been described in OSAHS [14]. As ongoing inflammatory responses play crucial roles in the progression of atherosclerosis which are definitely associated with higher cardiovascular and or cerebrovascular morbidity documented in OSAHS [15], increased serum levels of inflammatory cytokines in the atherosclerotic process, such as IL-6 and CRP, have been described in these patients. These cytokines are commonly raised in obesity, which is a confounding feature in patients with OSAHS [16]. On the contrary, some studies had not discovered an association between OSAHS and IL-6 and/or CRP levels [17,18]. Accordingly, there is disagreement about the relation between these inflammatory markers and OSAHS.

We found significant correlation between snoring, AHI, T90, and ODI 4\% with lymphocytic predominance in OPL, whereas CRP correlates significantly with neutrophils and eosinophil's in OPL in OSAHS group. High lymphocyte percentage was found in severe OSAHS, and it was correlated with AHI. Subsequently, the rise in inflammatory cells mainly lymphocytes in patients with OSAHS is correlated with the severity of OSAHS [8]. Yet, Salerno et al. [19] demonstrated the presence inflammation of the lower airway in OSAHS using induced sputum analysis which is a valid and commonly used procedure to assess the profile of bronchial inflammation. None of the patients were smokers nor had a history of acute or chronic pulmonary disease, and their sputum was characterized by variable grades of neutrophilic inflammation.

Inflammation associated with OSAHS was found likewise variable inflammatory biomarkers were measured mostly ILs and CRP. These markers were demonstrated and governed by obesity. There are synergistic influences of obesity and OSAHS on CRP along with reduction of CRP after treatment of OSAHS with CPAP [20]. Patients having OSAHS exhibit upper and lower inflammation of the airway. The cause of airway inflammation may be the mechanical stress on the mucosa produced by recurrent airway obstruction, nocturnal hypoxemia, and/or the associated systemic inflammation [21].Limitations of this study are that it is a single-center experience besides the relatively limited number of included patients, but this may be expected in prospective studies. Moreover, systemic inflammatory response was assessed solely by CRP. Addition of other markers of systemic inflammation like ILs or matrix metalloproteinase [22] would rather fortify the results of this study. Moreover, follow-up of our patients after positive airway pressure therapy would rather guarantee a full view of the link between OSAHS and the oropharyngeal as well as systemic inflammation.

\section{Conclusion}

This study has provided a novel vision into the pathophysiology of OSAHS in emphasizing the existence of upper airway lymphocytic inflammation. OPL is a simple, applicable, and easy noninvasive procedure for assessment of upper airway inflammation. Additional studies are required to settle our conclusions and to better describe the cellular pathogenesis in OSAHS. Further studies are essential to evaluate the clinical significance of airway inflammation in OSAHS, and the efficiency of the 
anti-inflammatory treatment on decreasing the OSAHS-induced inflammation of the airway and in revising the expected outcome of the disease. It is probable that using CPAP and reducing weight may diminish local and systemic inflammation.

\section{Financial support and sponsorship}

Nil.

\section{Conflicts of interest}

There are no conflicts of interest.

\section{References}

1 Entzian P, Linnemann K, Schlaak M, Zabel P. Obstructive sleep apnea and circadian rhythms of hormones and cytokines. Am J Respir Crit Care Med 1996; 153:1080-1086.

2 Hatipoğlu U, Rubinstein I. Inflammation and obstructive sleep apnea syndrome pathogenesis: a working hypothesis. Respiration 2003 70:665-671.

3 Sekosan M, Zakkar M, Wenig B, Olopade CO, Rubinstein I. Inflammation in the uvula mucosa of patients with obstructive sleep apnea. Laryngoscope 1996; 106:1018-1020.

4 Boyd JH, Petrof BJ, Hamid Q, Fraser R, Kimoff RJ. Upper airway muscle inflammation and denervation changes in obstructive sleep apnea. Am J Respir Crit Care Med 2004; 170:541-546.

5 Rubinstein I. Nasal inflammation in patients with obstructive sleep apnea. Laryngoscope 1995; 105:175-177.

6 Tauman R, Ivanenko A, O'Brien LM, Gozal D. Plasma C-reactive protein levels among children with sleep-disordered breathing. Pediatrics 2004; 113:564-569.

7 Larkin EK, Rosen CL, Kirchner HL, Storfer-Isser A, Emancipator JL, Johnson NL, et al. Variation of $\mathrm{C}$-reactive protein levels in adolescents: association with sleep-disordered breathing and sleep duration. Circulation 2005; 111:1978-1984.

8 Vicente E, Marin JM, Carrizo SJ, Osuna CS, González R, Marin-Oto M et al. Upper airway and systemic inflammation in obstructive sleep apnea. Eur Respir J 2016; 48:1108-1117.
9 Reid MB, Lännergren $\mathrm{J}$, Westerblad $\mathrm{H}$. Respiratory and limb muscle weakness induced by tumor necrosis factor-alpha: involvement of muscle myofilaments. Am J Respir Crit Care Med 2002; 166:479-484.

10 Huxtable AG, Vinit S, Windelborn JA, Crader SM, Guenther $\mathrm{CH}$, Watters $\mathrm{JJ}$, et al. Systemic inflammation impairs respiratory chemoreflexes and plasticity. Respir Physiol Neurobiol 2011; 178:482-489.

11 Hauber HP, Rüller S, Müller E, Hansen E, Zabel P. Pharyngeal lavage lymphocytosis in patients with obstructive sleep apnea: a preliminary observation. 2011. PLoS One 2011; 6:e16277.

12 Almendros I, Carreras A, Ramirez J, Montserrat JM, Navajas D, Farré R. Upper airway collapse and reopening induce inflammation in a sleep apnea model. Eur Respir J 2008; 32:399-404.

13 Li AM, Hung E, Tsang T, Yin J, So HK, Wong E, et al. Induced sputum inflammatory measures correlate with disease severity in children with obstructive sleep apnea. Thorax 2007; 62:75-79.

14 Devouassoux G, Lévy P, Rossini E, Pin I, Fior-Gozlan M, Henry M, et al. Sleep apnea is associated with bronchial inflammation and continuous positive airway pressure-induced airway hyper responsiveness. J Allergy Clin Immunol 2007; 119:597-603.

15 Pack Al, Gislason T. Obstructive sleep apnea and cardiovascular disease: a perspective and future directions. Prog Cardiovasc Dis 2009; 51:434-451.

16 Yokoe T, Minoguchi K, Matsuo H, Oda N, Minoguchi H, Yoshino G, et al. Elevated levels of C-reactive protein and interleukin-6 in patients with obstructive sleep apnea syndrome are decreased by nasal continuous positive airway pressure. Circulation 2003; 107:1129-1134

17 Taheri S, Austin D, Lin L, Nieto FJ, Young T, Mignot E. Correlates of serum C-reactive protein (CRP): no association with sleep duration or sleep disordered breathing. Sleep 2007; 30:991-996.

18 Arnardottir ES, Maislin G, Schwab RJ, Staley B, Benediktsdottir B Olafsson I, et al. The interaction of obstructive sleep apnea and obesity on the inflammatory markers C-reactive protein and interleukin6: the Icelandic Sleep Apnea Cohort. Sleep 2012; 35:921-932.

19 Salerno FG, Carpagnano E, Guido P, Bonsignore MR, Roberti A, Aliani M, et al. Airway inflammation in patients affected by obstructive sleep apnea syndrome. Respir Med 2004; 98:25-28.

20 Guilleminault $\mathrm{C}$, Kirisoglu C, Ohayon MM. C-reactive protein and sleepdisordered breathing. Sleep 2004; 27:1507-1511.

21 Chirinos JA, Gurubhagavatula I, Teff K, Rader TD, Wadden TA, Townsend $\mathrm{R}$, et al. CPAP, weight loss, or both for obstructive sleep apnea. $N$ Engl $J$ Med 2014; 370:2265-2275.

22 Youssef HA, Shalaby AO, Shaker OG, Sabry IM, Mahmoud EA. The role of matrix metalloproteinases 2 and 9 in obstructive sleep apnea. Egypt $J$ Bronchol 2014; 8:10-16. 